Research Paper

\title{
Hypobaric hypoxia preconditioning protects against hypothalamic neuron apoptosis in heat-exposed rats by reversing hypothalamic overexpression of matrix metalloproteinase-9 and ischemia
}

\author{
Chien-Ming Chao ${ }^{1,2}$, Chun-Liang Chen ${ }^{3}$, Ko-Chi Niü, Cheng-Hsien Lin ${ }^{5}$, Ling-Yu Tang6 ${ }^{6}$, Lieh-Sheng \\ Lin $^{7 凶}$, Ching-Ping Chang ${ }^{6 \bowtie}$ \\ 1. Department of Intensive Care Medicine, Chi Mei Medical Center, Liouying, Tainan, Taiwan. \\ 2. Department of Nursing, Min-Hwei College of Health Care Management, Tainan, Taiwan. \\ 3. Department of Gastroenterology and General Surgery, Chi Mei Medical Hospital, Chiali, Tainan, Taiwan. \\ 4. Department of Hyperbaric Oxygen, Chi Mei Medical Center, Tainan, Taiwan. \\ 5. Department of Medicine, Mackay Medical College, New Taipei City, Taiwan. \\ 6. Department of Medical Research, Chi Mei Medical Center, Tainan, Taiwan. \\ 7. Department of Neurosurgery, Taipei Medical University Hospital, Taipei, Taiwan.
}

$\square$ Corresponding author: Lieh-Sheng Lin M.D., D.M.S. Department of Neurosurgery, Taipei Medical University Hospital Mailing address: No. 250 Wu-Hsing Street, Taipei City 110, Taiwan; Phone: +886-2- 22490088; E-mail: liehsheng@gmail.com. Ching-Ping Chang, Ph.D., Department of Medical Research, Chi Mei Medical Center, Tainan, Taiwan Mailing address: No. 901, Zhonghua Rd., Yongkang District, Tainan City 710, Taiwan; Phone: +886-6-2812811; Fax: +886-6-2832639; E-mail: jessica.cpchang@gmail.com.

(c) The author(s). This is an open access article distributed under the terms of the Creative Commons Attribution License (https://creativecommons.org/licenses/by/4.0/). See http://ivyspring.com/terms for full terms and conditions.

Received: 2020.04.29; Accepted: 2020.08.24; Published: 2020.09.20

\begin{abstract}
Background: Hypoxia-inducible factor-l $\alpha$ (HIF-l $\alpha$ ), heat shock protein-72 (HSP-72), hemeoxygenase- 1 (HO-1), and matrix metalloproteinase-9 (MMP-9) have been identified as potential therapeutic targets in the brain for cerebral ischemia. To elucidate their underlying mechanisms, we first aimed to ascertain whether these proteins participate in the pathogenesis of heat-induced ischemic damage to the hypothalamus of rats. Second, we investigated whether hypobaric hypoxia preconditioning (HHP) attenuates heat-induced hypothalamic ischemic/hypoxic injury by modulating these proteins in situ.

Methods: Anesthetized rats treated with or without HHP were subjected to heat stress. Hypothalamic ischemic/hypoxic damage was evaluated by measuring hypothalamic levels of cerebral blood flow (CBF), partial oxygen pressure $\left(\mathrm{PO}_{2}\right)$, and hypothalamic temperature via an implanted probe. Hypothalamic apoptotic neurons were counted by measuring the number of NeuN/caspase-3/DAPI triple-stained cells. Hypothalamic protein expression of HIF-1 a, HSP-72, HO-1, and MMP-9 was determined biochemically.

Results: Before the start of the thermal experiments, rats were subjected to 5 hours of HHP (0.66 ATA or $18.3 \% \mathrm{O}_{2}$ ) daily for 5 consecutive days per week for 2 weeks, which led to significant loss of body weight, reduced brown adipose tissue (BAT) wet weight and decreased body temperature. The animals were then subjected to thermal studies. Twenty minutes after heat stress, heat-exposed rats not treated with HHP displayed significantly higher core and hypothalamic temperatures, hypothalamic MMP-9 levels, and numbers of hypothalamic apoptotic neurons but significantly lower mean blood pressure, hypothalamic blood flow, and $\mathrm{PO}_{2}$ values than control rats not exposed to heat. In heat-exposed rats, HHP significantly increased the hypothalamic levels of HIF-l $\alpha$, HSP-72, and HO-l but significantly alleviated body and hypothalamic hyperthermia, hypotension, hypothalamic ischemia, hypoxia, neuronal apoptosis and degeneration.

Conclusions: HHP may protect against hypothalamic ischemic/hypoxic injury and overexpression of MMP-9 by upregulating the hypothalamic expression of HIF-1a, HSP-72, and HO-1 in rats subjected to heatstroke.
\end{abstract}

Key words: hypothalamus; ischemic/hypoxic injury; heatstroke; hypobaric hypoxia 


\section{Introduction}

Heatstroke is characterized by hyperthermia, systemic inflammation, and multiple organ dysfunction syndromes, including arterial hypotension [1-3]. In particular, arterial hypotension and subsequent hypothalamic ischemic injury may be the main causes of heatstroke $[1,4]$.

In rodents, heat-induced thermoregulatory deficits and mortality may result from ischemic and oxidative damage to the hypothalamus (the essential thermoregulatory center in the mammalian brain). Compared to normothermic controls, mice subjected to heatstroke exhibit significantly higher levels of cellular ischemia markers (e.g., glutamate and the lactate-to-pyruvate ratio) and damage markers (e.g., glycerol) in the hypothalamus [5, 6]. In cerebral ischemia, enhanced expression of matrix metalloproteinase-9 (MMP-9) is associated with various complications, including neuronal damage, apoptosis, and blood-brain barrier (BBB) disruption [7]. Hemeoxygenase- 1 (HO-1) protects cells and tissues by exerting anti-inflammatory, antiapoptotic, antiproliferative, and antioxidative effects in response to noxious stimuli [8]. Hypoxia-inducible factor-1a (HIF-1a) acts as a potential therapeutic target for cerebral ischemia [9]. Heat shock protein-72 (HSP-72) overexpression appears to be critical for the development of thermotolerance and protection against heat-induced hypothalamic ischemic and oxidative damage in rodents. However, the interrelationships among these proteins in the hypothalamus during the pathogenesis of heatstroke remain unclear.

Changes associated with acclimation to hypoxia, including carotid body-mediated minute ventilation, which can improve oxygen saturation [10], diuresis, which can effectively increase hemoglobin and hematocrit concentrations, and stimulation of erythropoiesis, which can increase the number and oxygen-carrying capacity of red blood cells; occur within 1-3 weeks of high-altitude living (1500-3000 meters) or intermittent exposure to hypoxia [11]. These systemic responses have been experimentally linked to several downstream effects of HIF-1 $\alpha$, HSP-72 and other related proteins in hypothalamic tissues.

Hypobaric hypoxia preconditioning (HHP) is a transient phenomenon caused by brief episodes of sublethal hypoxia that induce various endogenous atrophic signals and robustly protect against subsequent lethal insults $[12,13]$. Although HHP has been shown to exert neuroprotective effects against ischemic brain injury [13, 14], it is still unknown whether HHP protects against heat-induced hypo- thalamic injury [15] by modulating hypothalamic proteins including HIF-a, MMP-9, HSP-72, and HO-1.

To address this question, the present study aimed to assess the effects of HHP on heat-induced ischemic and hypoxic damage to the rat hypothalamus. In addition, the temporal profiles of HIF-1 $\alpha$, HSP-72, HO-1, and MMP-9 in the hypothalamus were assessed in rats subjected to heatstroke and treated with or without HHP.

\section{Materials and Methods}

\section{Animal care}

All animal experiments were conducted on adult male Sprague-Dawley rats weighing 248-306 g. All experimental and animal care protocols were approved by the Institutional Animal Care and Use Committee of Chi Mei Medical Center, Tainan, Taiwan (approval no.: 106121110) in accordance with the guidelines of Guide for the Care and Use of Laboratory Animals published by the US National Institutes of Health [16], and care was taken to minimize pain and suffering. The rats were housed in a temperature-controlled room $\left(24 \pm 1.0^{\circ} \mathrm{C}\right)$ with a relative humidity of $50 \pm 10 \%$ on a $12 / 12$ hour-light/ dark cycle, and food pellets and water were provided ad libitum.

\section{Physiological variable monitoring}

Each animal was anesthetized with sodium pentobarbital $(40 \mathrm{mg} / \mathrm{kg}$ body weight, i.p.), and the femoral artery was cannulated with polyethylene tubing to measure mean arterial blood pressure (MABP) and heart rate (HR). Body core temperature (Tco) was monitored continuously by a thermocouple inserted into the rectum, and MABP and HR were continuously monitored with a pressure transducer. The head of the animal was mounted on a stereotaxic apparatus (David Kopf Instruments, Tujunga, CA, USA) with the nose bar positioned $3.3 \mathrm{~mm}$ below the horizontal line. Following a midline incision, the skull was exposed, and a burr hole was made in the skull to allow insertion of a $100-\mu \mathrm{m}$-diameter thermocouple and two $230-\mu \mathrm{m}$ fibers attached to an oxygen probe. This combined probe was used to measure oxygen, temperature, and microvascular blood flow. OxyLite and OxyFlo instruments were used for the measurements. The OxyLite 2000 instrument (Oxford Optronix, Oxford, UK) is a two-channel device (measures partial oxygen pressure $\left[\mathrm{PO}_{2}\right]$ and temperature at 2 locations simultaneously), whereas the OxyFlo 2000 instrument is a two-channel laser Doppler perfusion monitoring instrument. Together, these 2 instruments simultaneously provided tissue blood flow, oxygenation, and temperature data [17]. 


\section{HHP simulation}

Animals in the HHP groups were exposed to 5 hours of simulated HHP of $4000 \mathrm{~m}$ in a hypobaric chamber $\left(0.66\right.$ ATA or $\left.18.3 \% \mathrm{O}_{2}\right)$ daily for 5 consecutive days per week for 2 weeks before the start of heat exposure [18]. All animals in the non-HPP groups were exposed to 1.0 ATA $\left(21 \% \mathrm{O}_{2}\right)$ for the equivalent period of time.

\section{Induction of heatstroke}

The Tco of the normothermic control group was maintained at about $37^{\circ} \mathrm{C}$ with a folded heating pad at a room temperature of $26^{\circ} \mathrm{C}$ throughout the entire experiment. For the heatstroke group, the heating pad was removed before the start of heat stress (ambient temperature [Ta] of $42^{\circ} \mathrm{C}$ with a relative humidity of $60 \%$ ). In the heatstroke group, an MABP value of 25 $\mathrm{mmHg}$ below the peak accompanied by excessive body hyperthermia (i.e., $\sim 41.5{ }^{\circ} \mathrm{C}$ Tco and $\sim 40.0{ }^{\circ} \mathrm{C}$ hypothalamic temperature) was observed approximately $70 \mathrm{~min}$ after the initiation of heat stress; this time point was arbitrarily used as the onset of heatstroke [19]. Heat was withdrawn from the heat-exposed animals $70 \mathrm{~min}$ after heat stress, and the animals were allowed to recover at room temperature $\left(26^{\circ} \mathrm{C}\right)$.

\section{Experimental groups}

The animals were randomly assigned randomly to one of the following four groups: the non-HHP + non-heat-exposed group, HHP + non-heat-exposed group, non-HP + heat-exposed group, and HHP + heat-exposed group. In the non-heat-exposed groups, the Tco was maintained at approximately $37^{\circ} \mathrm{C}$ with a heating pad. The animals in the heat-exposed groups were exposed to heat stress $\left(40^{\circ} \mathrm{C}\right)$ for exactly $70 \mathrm{~min}$.

\section{Western blot analysis}

The rats were anesthetized and underwent intracardiac perfusion with 0.1 M PBS ( $\mathrm{pH} 7.4$ ) [20, 21]. The hypothalamic region of the brain was rapidly isolated, and the brain tissues were homogenized using a homogenizer. Total protein was extracted using protein extraction reagent (Bio-Rad Laboratories, CA, USA), and the protein concentration was quantified by using the Bradford method (Bio-Rad protein assay kit, Bio-Rad Laboratories). The protein samples $(50 \mu \mathrm{g})$ were separated by $20 \%$ sodium dodecyl sulfatepolyacrylamide gel electrophoresis and were subsequently transferred onto polyvinylidene fluoride membranes. The blots were blocked and incubated with a primary antibody specific for HSP-72, HIF-1a or HO-1. The membranes were then incubated with horseradish peroxidase-conjugated anti-rabbit immunoglobulin G and anti-mouse IgG. Following secondary antibody incubation, the immunoblots were visualized with an enhanced chemiluminescence detection system. The expression of HSP-72, HIF-1a, HO-1 and $\beta$-actin was semiquantified using a gel densitometry scanning program.

\section{Gelatin zymography}

The levels of MMP-9 in the brain homogenates were measured by gelatin zymogram following a previously described technique [22]. The brains were quickly removed from the rats under general anesthesia, and the tissues were frozen immediately in liquid nitrogen and stored at $-80^{\circ} \mathrm{C}$. Total protein concentrations were determined using the bicinchoninic acid protein assay. Protein samples (50 $\mu \mathrm{g}$ ) were prepared, loaded on $10 \%$ tris-glycine gels with $0.1 \%$ gelatin as a substrate and separated. After electrophoresis and incubation, the gels were stained with $3 \%$ methanol, $10 \%$ acetic acid, and $0.5 \% \mathrm{wt} / \mathrm{vol}$ Coomassie brilliant blue for 2 hours and then destained. Gelatinolytic activity was manifested as horizontal white bands on a blue background. MMP bands were identified after standard techniques using molecular weight criteria and a human MMP-9 control, which was purchased from Chemicon [23].

\section{Hematoxylin and Eosin (H\&E) staining}

We evaluated brain tissue damage following a previously described method [24]. At the end of the experiments, the rats were anesthetized as described above and intracardially perfused with isotonic chloride solution followed by $4 \% \quad(\mathrm{w} / \mathrm{v})$ paraformaldehyde (Bio-Rad Laboratories) in $0.1 \mathrm{M}$ sodium phosphate buffer ( $\mathrm{pH}$ 7.4; Bio-Rad Laboratories). The brains were subsequently removed, fixed for 48 hours in $4 \%(\mathrm{w} / \mathrm{v})$ paraformaldehyde, and embedded in paraffin (Bio-Rad Laboratories), and brain tissues were sliced into 4- $\mu \mathrm{m}$ coronal sections and stained with hematoxylin-eosin (H\&E) (Sigma-Aldrich, MA, USA).

Semiquantitative histological analysis was performed after H\&E staining to evaluate morphological changes in neural cells and the severity of damage to the brain following heat stress. Brain sections (corresponding to coronal coordinates of Paxinos and Watson [25] at bregma -2.2 -3.6 mm, lateral $0 \sim 1 \mathrm{~mm}$, and depth $8.0 \sim 10 \mathrm{~mm}$ ) were semiquantitatively scored [26] from 0 to $4(0=$ no detectable lesion, $1=$ lesion involving $<25 \%$ of the field, 2 = lesion involving $25 \%-50 \%$ of the field, $3=$ lesion involving $50 \%-75 \%$ of the field, and $4=$ lesion involving $>75 \%$ of the field). Each hypothalamus region was evaluated for morphological changes as 
follows [27]: $0=$ normal morphology, $1=$ minor damage (edema and few pyknotic cells), $2=$ moderate damage (structural disorganization, edema, many pyknotic cells, and vacuolization), and $3=$ intense damage (structural disorganization, edema, many pyknotic cells, and vacuolization). Brain damage was scored and evaluated, and the scores were multiplied to yield final scores, which ranged from 0 to 12 , for each animal. Representative images were collected.

\section{Neuronal apoptosis}

Coronal sections were incubated with 10\% normal donkey serum, primary antibodies (rabbit anti-rat neuron-specific nuclear protein [NeuN; 1:200, Santa Cruz Biotechnology] and rabbit anti-cleaved caspase-3 [1:100; Santa Cruz Biotechnology]) and fluorescent-labeled secondary antibodies (Alexa Fluor 568-conjugated goat anti-rabbit IgG, Alexa Fluor 568-conjugated goat anti-mouse IgG or Alexa Fluor 488-conjugated goat anti-mouse; Santa Cruz Biotechnology). The sections were incubated with 4,6-diamidino-2-phenylindole (DAPI, Vectashield ${ }^{\circledR}$ Vector Laboratories, Burlingame, CA, USA) to counterstain the nuclei. Another section was stained with a terminal deoxyribonucleotide transferasemediated dUTP nick end labeling (TUNEL) assay kit (\#630108, Clontech, Palo Alto, CA, USA) to quantify neuronal apoptosis. The sections were subsequently washed with phosphate buffer, and the nuclei were counterstained with DAPI.

\section{Determination of hypothalamic glutamate levels, glycerol levels and lactate-to-pyruvate ratio}

To measure hypothalamic glutamate concentrations, glycerol concentrations, and lactateto-pyruvate ratio, aliquots of samples were injected into a CMA600 microdialysis analyzer (Carnegie Medicine, Stockholm, Sweden) [28].

\section{Data presentation and statistical analysis}

The data are presented as the means \pm SDs ANOVA was used to analyze factorial experiments, and Duncan's multiple range test was used for post hoc comparisons among means. GraphPad Prism (version 7.01 for Windows; GraphPad Software, San Diego, CA, USA) was used for these analyses. A $p$-value of less than 0.05 was considered statistically significant.

\section{Results}

\section{HHP reduces body weight, brown adipose tissue (BAT) weight and temperature}

As shown in Table 1, after 2 weeks, the average body weight of HHP-treated rats was significantly lower than that of untreated rats, which remained unchanged. Both the BAT temperature and weight values of the HHP group were significantly lower than those of the non-HHP group (Table 1).

\section{HHP attenuates heat-induced hyperthermia, hypotension and hypothalamic ischemia/ hypoxia}

Compared to the non-HHP + non-heat-exposed group, the non-HHP + heat-exposed rats exhibited significant increases in Tco (Fig. $1 \mathrm{~B} ; \sim 42^{\circ} \mathrm{C}$ vs. $\sim 37^{\circ} \mathrm{C}$ ) and hypothalamic temperature (Fig. $1 \mathrm{C} ; \sim 41{ }^{\circ} \mathrm{C}$ vs. $\sim 37^{\circ} \mathrm{C}$ ) but significant decreases in MABP (Fig. $1 \mathrm{D}$; $\sim 20 \mathrm{mmHg}$ vs. $\sim 90 \mathrm{mmHg}$ ), HR (Fig. 1E; 300 beats/min vs. $\sim 450$ beats/min), hypothalamic cerebral blood flow (CBF) (Fig. 1F; 50\% vs. 100\%), and hypothalamic $\mathrm{PO}_{2}$ (Fig. 1G; $\sim 15 \mathrm{mmHg}$ vs. 22 $\mathrm{mmHg}$ ) $20 \mathrm{~min}$ after heatstroke (or $90 \mathrm{~min}$ after the start of 70 min of heat stress; Fig. 1A). Heat-induced hypotension and hypothalamic ischemia/hypoxia were significantly attenuated by HHP, as shown in the $\mathrm{HHP}+$ heat-exposed rats (MABP, 80 $\mathrm{mmHg}$; $\mathrm{CBF}, \sim 120 \%$; $\mathrm{PO}_{2}, \sim 30 \mathrm{mmHg}$ ). Biochemical assays also confirmed that cellular ischemia indicators, namely, glutamate levels and the lactate/pyruvate ratio, and a cellular damage indicator, namely, glycerol levels (Fig. 2), were significantly increased in the hypothalamus by heat stress. HHP significantly attenuated the increases in these cellular ischemia and injury indicators in the hypothalamus (Fig. 2).

\section{$H H P$ reduces heat-induced increases in hypothalamic cell damage scores and the number of hypothalamic apoptotic neurons}

H\&E staining revealed that the non-HHP + heat-exposed group exhibited significantly higher hypothalamic cell damage scores than the non-HHP + non-heat-exposed group and the HHP + non-heat-exposed group (0 vs. 2.5) (Fig. 3). Both the non-HHP+non-heat-exposed and the HHP+nonheat-exposed groups exhibited almost normal morphology, whereas in the non-HHP+heat-exposed group, $\sim 50 \%$ of the field showed moderate damage (e.g., structural disorganization, edema, many pyknotic cells, and vacuolization). Although HHP did not significantly affect the morphology of the paraventricular region in the hypothalamus in nonheat-exposed rats, it significantly attenuated the increase in hypothalamic cell damage scores in heatexposed rats (Fig. 3). Immunofluorescence staining further demonstrated that the non-HHP+heatexposed rats exhibited significantly more NeuN+ caspase-3+DAPI-positive cells (Fig. 4) and NeuN+TUNEL+DAPI-positive cells (Fig. 5) in the hypothalamus than the non-HHP+non-heat-exposed 
and HHP+non-heat-exposed rats. Although HHP did not affect the number of either NeuN+caspase3+DAPI-positive cells (Fig. 4) or NeuN+TUNEL+ DAPI-positive cells in the hypothalamus (Fig. 5), it

(A)

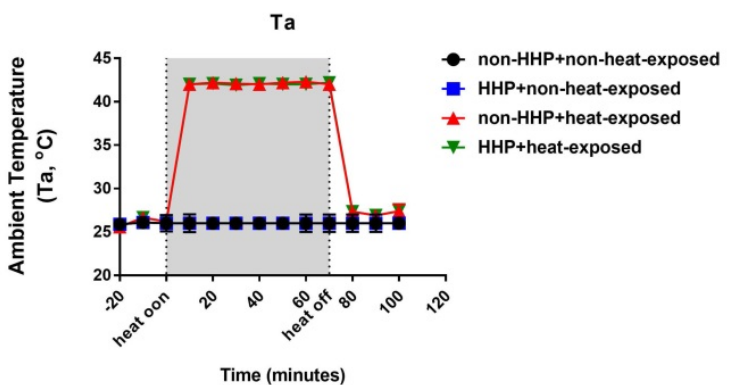

(B)

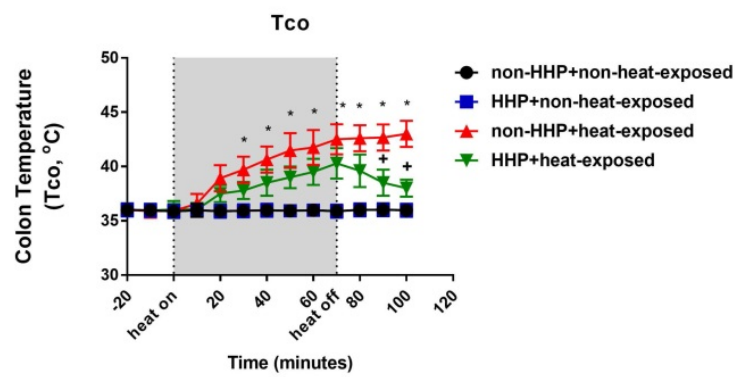

(C)

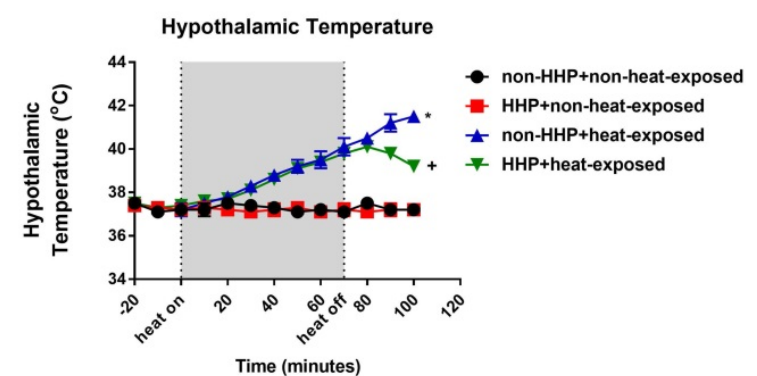

(D)

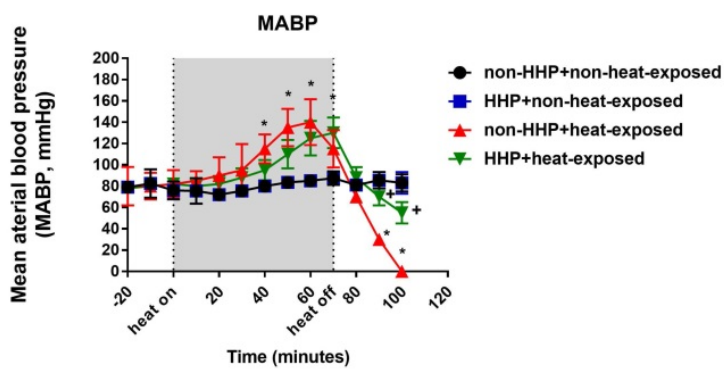

significantly prevented the increase in the number of hypothalamic apoptotic neurons following heat exposure (Fig. 4 and Fig. 5).

(E)

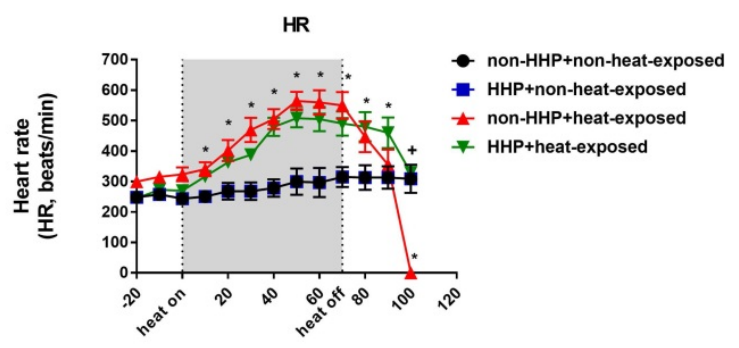

Time (minutes)

(F)

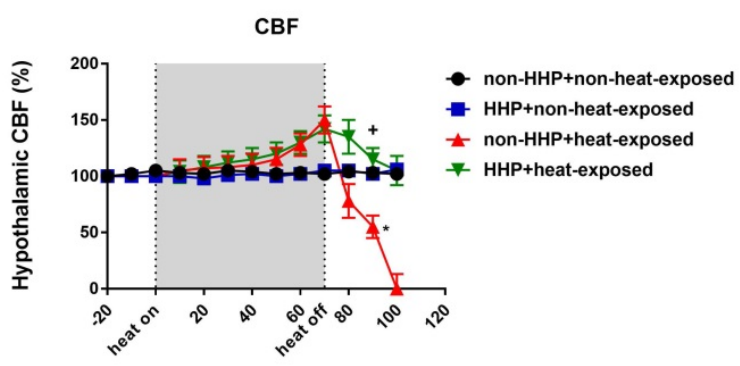

Time (minutes)

(G)

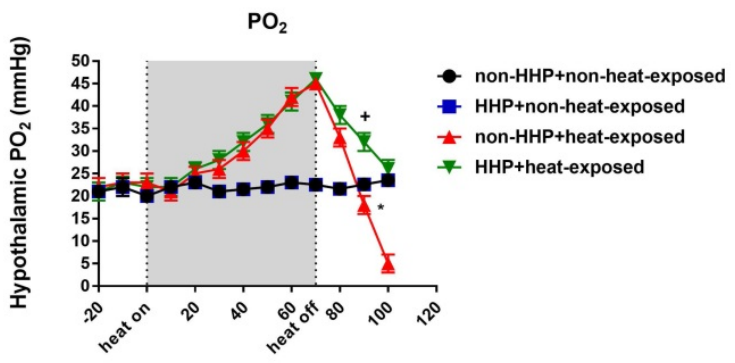

Time (minutes) 
(A)

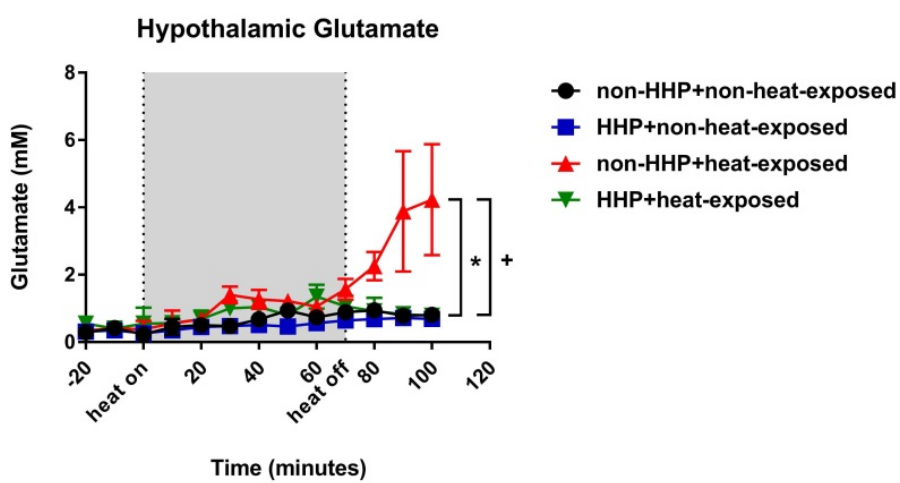

(B)

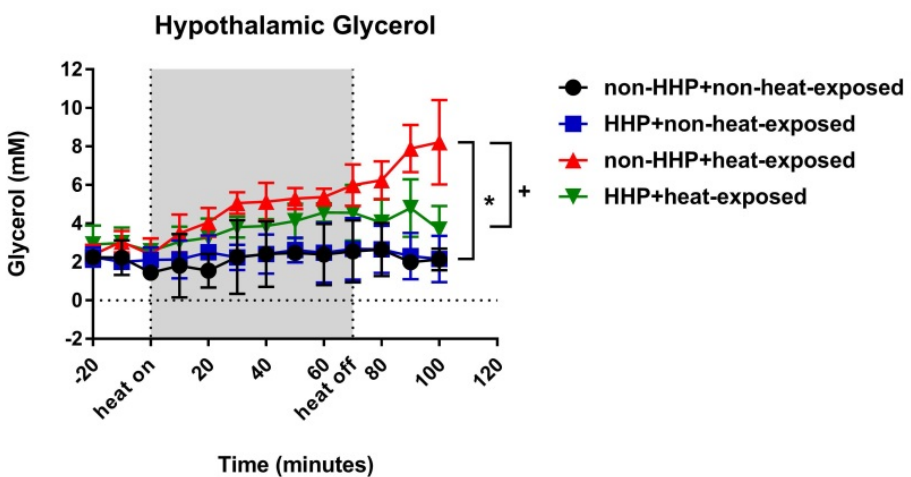

(C)

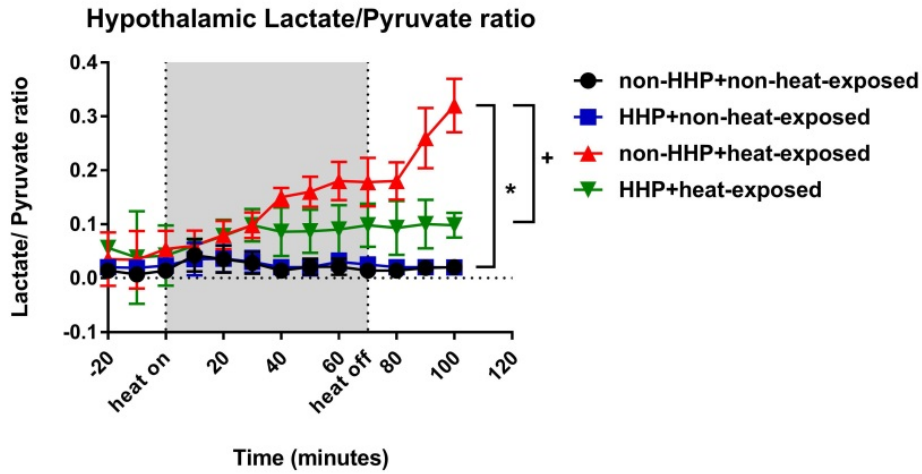

Figure 2. Changes in hypothalamic (A) glutamate levels, (B) glycerol levels, and (C) lactate/pyruvate ratio in non-HHP+non-heat-exposed rats ( $)$, HHP + non-heat-exposed rats $(\boldsymbol{\square})$, non-HHP + heat-exposed rats $(\boldsymbol{\Delta})$ and HHP + heat-exposed rats $(\boldsymbol{\nabla})$ over time. The values are the means \pm SDs; $\mathrm{n}=10$ rats per group. $* P<0.05$ vs. non-HHP+non-heat-exposed group; $+P<0.05$ vs. non-HHP+heat-exposed group.

Table 1. Means and standard deviations (SDs) of body weight, BAT weight and BAT temperature in the two experimental groups ( $n=6$ per group)

\begin{tabular}{lll}
\hline & Non-HHP & HHP \\
\hline Initial body weight $(\mathrm{g})$ & $274 \pm 44$ & $275 \pm 45$ \\
Final body weight $(\mathrm{g})$ & $277 \pm 42$ & $255 \pm 43$ \\
Initial - final weight $(\triangle$ weight; $\mathrm{g})$ & $30 \pm 6$ & $-17 \pm 7^{*}$ \\
BAT weight $(\mathrm{g})$ & $0.59 \pm 0.08$ & $0.42 \pm 0.06^{*}$ \\
BAT temperature $\left({ }^{\circ} \mathrm{C}\right)$ & $35.8 \pm 0.02$ & $35.5 \pm 0.03$ \\
\hline
\end{tabular}

${ }^{*} p<0.05$, HHP vs. non-HHP;

$\mathrm{HHP}$, hypobaric hypoxic preconditioning; BAT, brown adipose tissue.

\section{HHP increases the hypothalamic levels of HSP-72, HIF-1 $\alpha$, and HO-1 in heat-exposed} rats

Western blot analysis revealed no significant differences in the hypothalamic levels of HSP-72, HIF-1 $\alpha$, and HO-1 between the non-HHP+non-heatexposed group and the non-HHP+heat-exposed group ( $P>0.05$; Fig. $6 \mathrm{~A}$ and Fig. $6 \mathrm{~B})$. On the other hand, the HHP+heat-exposed rats exhibited significantly higher hypothalamic levels of HSP-72, HIF- $1 \alpha$, and HO-1 than the non-HHP+heat-exposed rats $(P<0.05$; Fig. $6 \mathrm{~A}$ and Fig. $6 \mathrm{~B})$. 


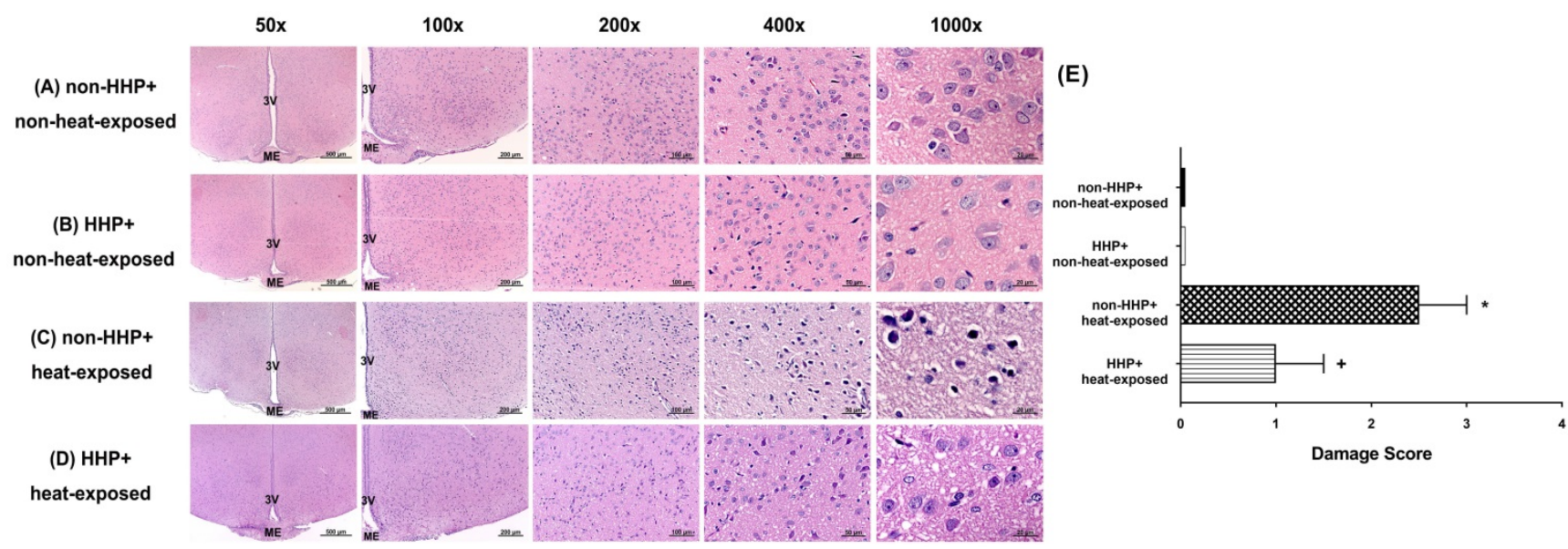

Figure 3. (A) Photomicrographs of hypothalamic hematoxylin-eosin staining of a non-HHP + non-heat-exposed rat (A), an HHP + non-heat-exposed rat (B), a non-HHP + heat-exposed rat (C), and an HHP + heat-exposed rat (D). Images of brain morphology are shown at 50x, 100x, 200x, 400x and 1000x magnification. Scale bars= 500 $\mu \mathrm{m}, 200$ $\mu \mathrm{m}, 100 \mu \mathrm{m}, 50 \mu \mathrm{m}$, and $20 \mu \mathrm{m}$. Rats in the heat-exposed groups were killed 90 min after heat stress, and rats in the non-heat-exposed groups were skilled at the equivalent time. (E) The hypothalamic damage scores of the different groups are presented as the means $\pm S D s ; n=10$ per group. $* P<0.05$ for the non-HHP + heat-exposed group versus the non-HHP + non-heat-exposed group; $+P<0.05$ for the HHP + heat-exposed group versus the non-HHP + heat-exposed group. $3 \mathrm{~V}=$ third ventricle; $M E=$ median eminence.
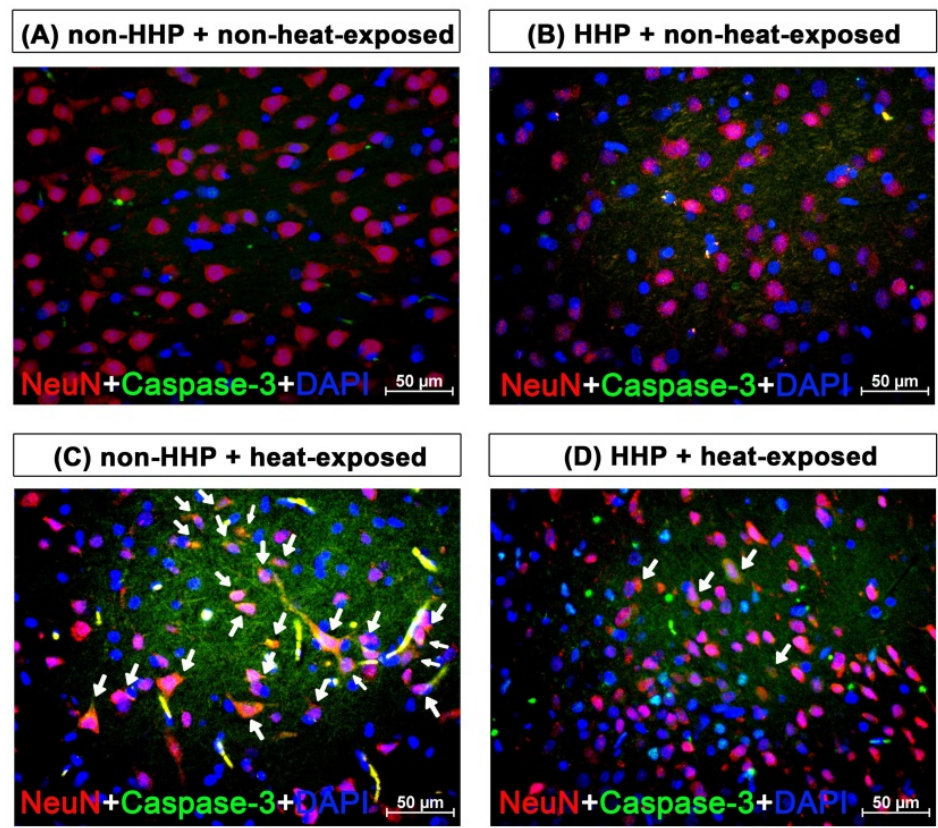

(E)

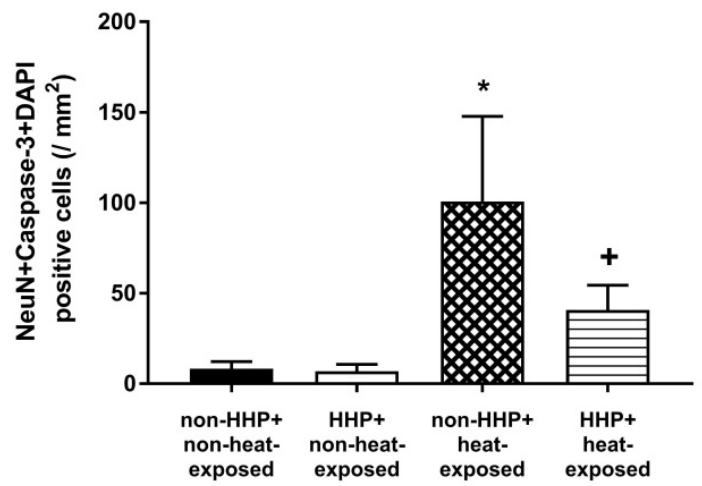

Figure 4. Photomicrographs of immunofluorescence staining of hypothalamic apoptotic neurons (NeuN+caspase-3+DAPI-positive cells) in a non-HHP + non-heat-exposed rat (A), an HHP + non-heat-exposed rat (B), a non-HHP+heat-exposed rat (C), and an HHP+heat-exposed rat (D). The animals were killed 90 min after heat stress or at the equivalent time for the non-heat-exposed groups. Scale bar $=50 \mu \mathrm{m}$. (E) The values are presented as the means $\pm S D s ; n=10$ per group. $* P<0.05$ for the non-HHP + heat-exposed group versus the non-HHP + non-heat-exposed group; $+P<0.05$ for the HHP + heat-exposed group versus the non-HHP + heat-exposed group. 


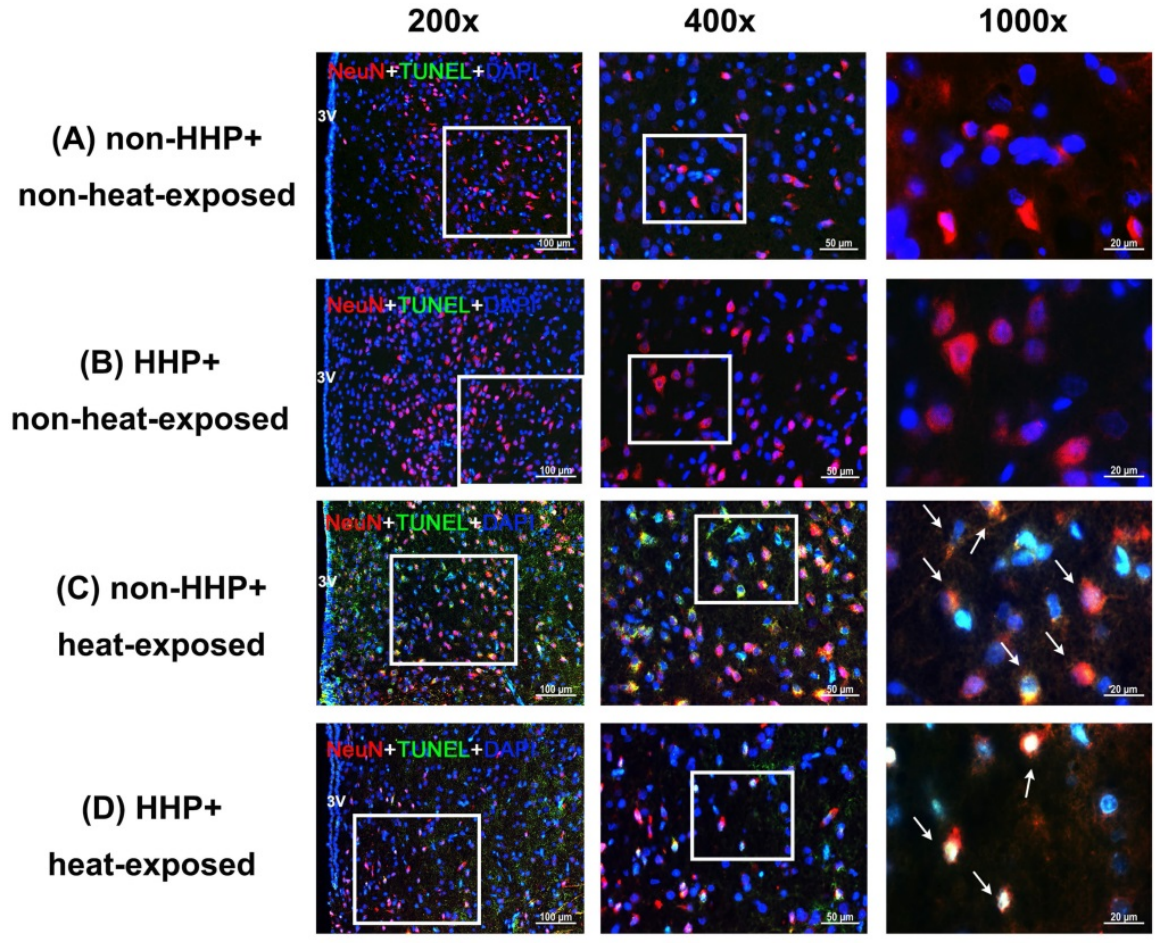

$(\mathrm{E})$

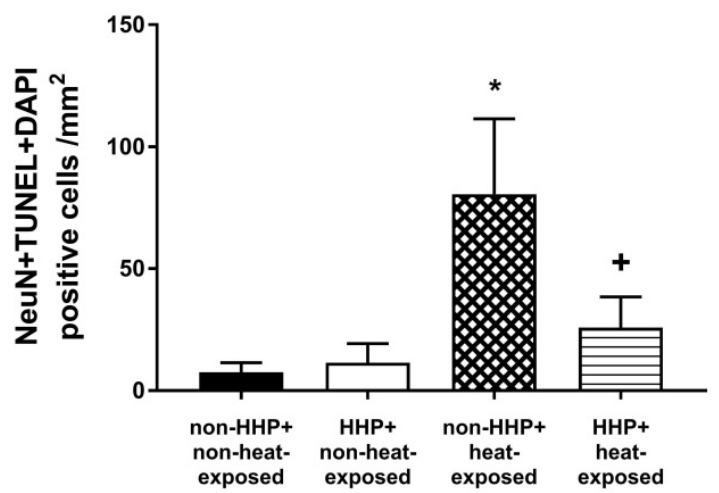

Figure 5. Photographs of immunofluorescence staining of hypothalamic apoptotic neurons (NeuN+TUNEL+DAPI-positive cells) in a non-HHP+non-heat-exposed rat (A), an $\mathrm{HHP}+$ non-heat-exposed rat (B), a non-HHP+ heat-exposed rat (C), and an HHP+ heat-exposed rat (D). The animals were killed 90 min after heat stress or at the equivalent time for the non-heat-exposed groups. Scale bar $=50 \mu \mathrm{m}$ ). (E) The values are presented as the mean $\pm S D s ; n=10$ per group. $* P<0.05$ for the non-HHP+heat-exposed group versus the non-HHP+non-heat-exposed group; $+P<0.05$ for the HHP+heat-exposed group versus the non-HHP+heat-exposed group.

\section{HHP decreases heat-induced MMP-9 over- expression and activity in heat-exposed rats}

Gelatin zymography analysis revealed that the non-HHP+heat-exposed group exhibited significantly higher levels of MMP-9 activity in the hypothalamus than the non-HHP+non-heat-exposed group and the $\mathrm{HHP}+$ non-heat-exposed group (Fig. 6C and Fig. 6D). However, the HHP+heat-exposed rats exhibited significantly lower hypothalamic MMP-9 activity than the non-HHP+heat-exposed rats (Fig. 6C and Fig. 6D).

\section{Discussion}

In the present study, which used an experimental rat model of heatstroke, heatstroke- exposed animals displayed hyperthermia (i.e., Tco of $42^{\circ} \mathrm{C}$ ), arterial hypotension (i.e., MABP of $20 \mathrm{mmHg}$ ), bradycardia (i.e., HR of $\sim 200$ beats/min), hypothalamic ischemia (i.e., $\mathrm{CBF}$ of $40 \%$ of the original level), hypoxia (i.e., $\mathrm{PO}_{2}$ of $\sim 5 \mathrm{mmHg}$ ), neuronal degeneration (i.e., increased neuronal damage scores) and apoptosis (i.e., increased numbers of Neu-N/caspase-3/DAPI-positive cells and NeuN+TUNEL+DAPI-positive cells). In addition, heat-induced hypothalamic ischemic/hypoxic injury were associated with increased hypothalamic levels of MMP-9 activity. The most striking finding of the present study was that HHP protected against heat-induced hypotension (e.g., increased MABP to $\sim 60 \mathrm{mmHg}$ ), bradycardia (e.g., increased HR to $\sim 350$ 
beats/min), hypothalamic ischemia (e.g., increased CBF to $\sim 100 \%$ ), hypoxia (increased the $\mathrm{PO}_{2}$ to 17 $\mathrm{mmHg}$ ), hypothalamic neuronal degeneration (e.g., decreased neuronal damage scores) and apoptosis (e.g., reduced the number of apoptotic neurons) and increased hypothalamic levels of MMP-9 activity. Simultaneously, HHP increased the hypothalamic expression of HSP-72, HIF-1a, and HO-1 in heat-exposed animals. Although HHP did not affect heat-induced hyperthermia, it attenuated heatinduced hypothalamic overexpression of MMP-9 as well as hypothalamic ischemia/hypoxia and its complications (e.g., hypothalamic neuronal degeneration and apoptosis).

HIF-1a maintains oxygen homeostasis and facilitates cellular adaptation to low oxygen conditions by regulating many downstream genes that participate in angiogenesis, erythropoiesis, energy metabolism, apoptosis and neuronal stem/progenitor cell proliferation [9]. Heat acclimation (HA) confers protection against acute heat stress and delays thermal injury [29, 30]. Increased levels of HIF-1a as well as HSP72 and its metabolic targeted genes are among the cytoprotective changes that occur during HA [31]. HIF-1a, the master regulator of oxygen homeostasis, participates in the
(A)

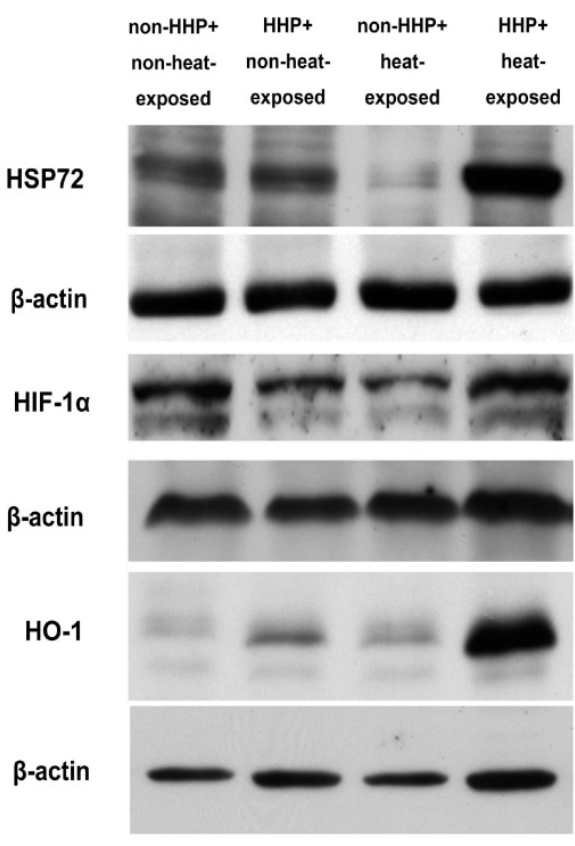

(B)

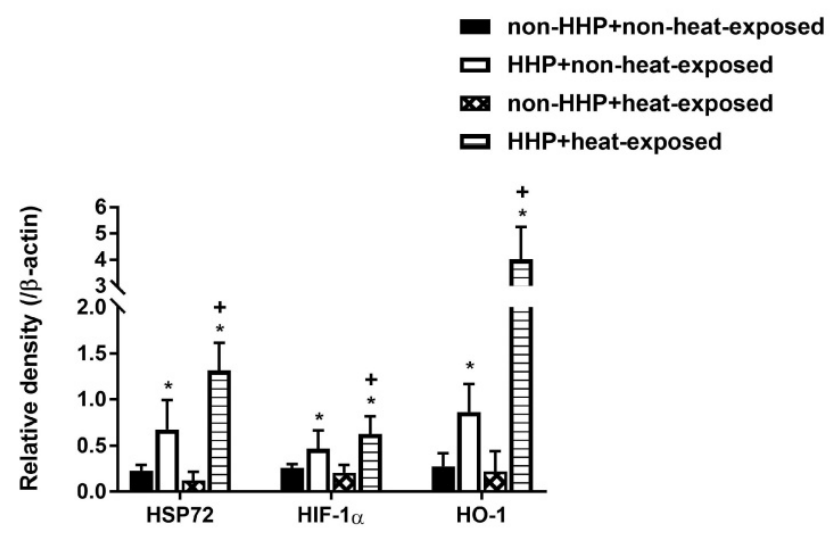

(D)

(C)

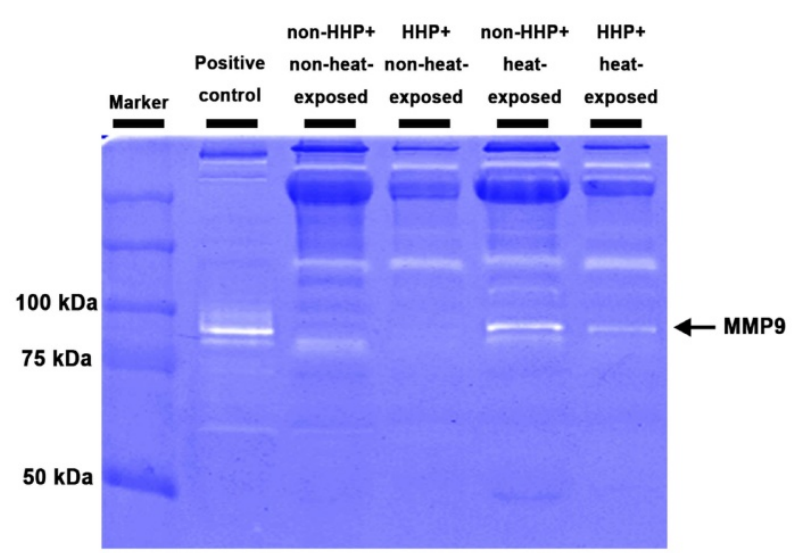

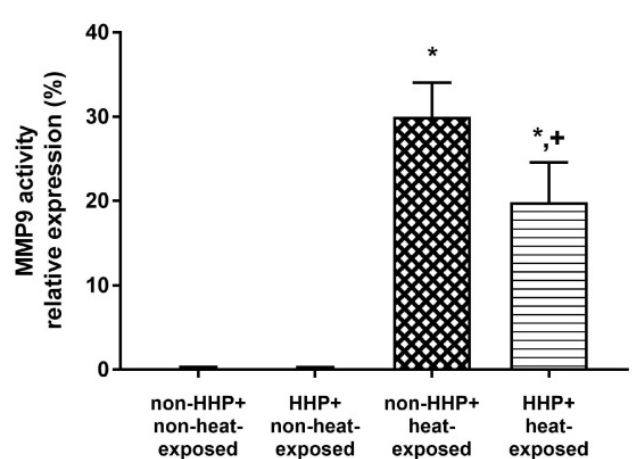

Figure 6. (A) Western blot analysis of HSP72, HIF-1 $\alpha$ and HO-1 in hypothalamic tissues from a non-HHP + non-heat-exposed rat, an HHP + non-heat-exposed rat, a non-HHP + heat-exposed rat, and an HHP + heat-exposed rat. (B) The fold-change values represent the mean of 10 samples $(n=10)$ divided by the mean of the 10 controls ( $n=10)$. (C) Gelatin zymography analysis of MMP-9 in hypothalamic tissues from a non-HHP + non-heat-exposed rat, an HHP + non-heat-exposed rat, a non-HHP + heat-exposed rat, and an HHP + heat-exposed rat. (D) The percent expression values represent the mean of 10 samples $(n=10)$. The results are presented as the means $\pm S D s * P<0.01$ compared with the non-HHP and non-heat-exposed groups; $+P<0.01$ compared with the non-HHP + heat-exposed group. 
process of HA $[32,33]$. Our present data show that HHP exerts the same cytoprotective effects as HA. HHP may protect against heat-induced hypothalamic injury by upregulating HIF-1a. In contrast, our present results are not consistent with several previous findings. Neuron-specific HIF-1a-deficient mice display reductions in both the infarct volume and expression of proapoptotic genes following bilateral common carotid artery occlusion [34]. In addition, early inhibition of HIF-1a with small interfering RNA reduces ischemia-reperfusioninduced brain injury in rats [35]. Inhibition of HIF-1a by HIF-1a inhibitors such as 2-methoxyestradiol [35], mir-335 [9] and hyperbaric oxygen [36] in the acute phase of ischemic stroke exerts beneficial effects via inhibition of apoptotic genes. Thus, downregulating HIF-a expression in the early period of cerebral ischemia could be efficacious in reducing cerebral ischemic damage. The reason for the discrepancy between our results and the results of these previous studies is currently unclear.

Transgenic mice have been used to examine the role of HSP72 in experimental heatstroke [6]. Compared to transgene-negative littermate controls ([-]HSP72 mice), transgenic mice heterozygous for the porcine HSP-72 $\beta$ gene ([+]HSP 72 mice) exhibit lower levels of cellular ischemia, inflammatory and oxidative damage markers in the hypothalamus and lower neuronal damage scores 4 hours after heat stress. [-]HSP72 mice were subjected to heat stress, and 4 hours after exposure, none of the 12 mice were still living, and the Tco of the mice was $34.2 \pm 0.4{ }^{\circ} \mathrm{C}$. When [+]HSP-72 mice were exposed to the same heat treatment, the fraction survival and Tco values were significantly increased to $12 / 12$ and $37.4 \pm 0.3{ }^{\circ} \mathrm{C}$, respectively. This study indicates that HSP72 overexpression appears to be critical for the development of thermotolerance and protection from heat-induced hypothalamic ischemic and oxidative damage. In the present study, we further confirmed that HHP-induced HSP72 overexpression in the hypothalamus protects against ischemic/hypoxic injury in the hypothalamus in heat-exposed rats. In studies of cerebral ischemia, neurodegenerative disease, epilepsy, and trauma, HSP72 has been shown to be neuroprotective [37]. Proteins that accumulate in Alzheimer's disease models are able to be degraded in HSP72-overexpressing transgenic mice, leading to neurological improvements [38].

Hemeoxygenase-1 (HO-1) is an inducible isoform of the first and rate-limiting enzyme of the degradation of heme into iron, carbon monoxide, and biliverdin. It possesses anti-inflammatory, antiapoptotic, angiogenic, and cytoprotective functions, suggesting that it plays potential protective and defensive role in several pathophysiological states, including ischemic stroke [39]. HO-1 knockout mice exhibit greater ischemic damage than wild-type mice [40]. Induction of HO-1 expression with acetyl-11keto- $\beta$-boswellic acid [41] or saponins [42] effectively reduces stroke- or reperfusion-induced injury. These observations provide compelling information that $\mathrm{HO}-1$ activation contributes to neuroprotection under many conditions. HO-1 exerts its anti-inflammatory effects by upregulating the expression of interleukin-10 (an anti-inflammatory cytokine) and interleukin-1 receptor antagonist [43]. In the present study, HHP may have attenuated hypothalamic inflammation and ischemic/hypoxic injury by enhancing HO-1 expression in the hypothalamus.

MMP-9 plays several important roles associated with the actions of neutrophils, such as degradation of extracellular matrix, activation of IL-1 $\beta$, and cleavage of several chemokines [44]. In a mouse model, MMP-9 deficiency results in resistance to endotoxin shock, suggesting that MMP-9 is important in sepsis [45]. Knockout of MMP 9 results in delayed apoptosis, vascularization, and ossification of hypertrophic chondrocytes [46]. Enhanced MMP-9 expression and activity have been observed in many neuropsychiatric disorders and central nervous system disorders, including ischemic stroke [28, 47, 48]. In cerebral ischemia, enhanced expression of MMP 9, which is associated with various complications, including excitotoxicity, neuronal damage, apoptosis, and BBB opening, which leads to cerebral edema and hemorrhagic transformation, is observed [7]. It is evident that brain ischemia, inflammation, and oxidative damage rather than hyperthermia of the body are the main causes of heatstroke [1, 4]. In addition, heatstroke resembles septic shock in many ways $[1,4]$. In the present study, HHP may have inhibited the overexpression of MMP-9 in the hypothalami of rats subjected to heatstroke, resulting in a reduction in hypothalamic ischemia, hypoxia, neuronal degeneration and apoptosis.

Hypoxia-inducible factors are key molecules that regulate the cellular response to hypoxia and inflammation and play a major role in normal cell function and survival [49]. Current evidence also indicates that the HIF-1 $\alpha$ pathway in the hypothalamus may be involved in the regulation of food intake and energy expenditure [49]. Previous studies have shown that 30 cycles of intermittent hypoxia per hour for 8 hours per day for 21 days reduces body weight and BAT wet weight in rats [50]. In our present study, we further confirmed that five hours of HHP (0.66 ATA or $18.3 \% \mathrm{O}_{2}$ ) daily for 5 consecutive days per week for 2 weeks before the start of heat exposure caused both weight loss and reduced 
BAT wet weight in rats. BAT activation occurs at temperatures between 4 and $16{ }^{\circ} \mathrm{C}$ [51]. In our present study, all HHP experiments were conducted at $26^{\circ} \mathrm{C}$. It is, therefore, improbable that procedure-induced BAT behavior or uncoupling protein 1 function influenced the protective effects of HHP in heatstroke.

Fig. 7A depicts core the molecular networks identified by ingenuity pathway analysis (IPA) [5]. The altered proteins, including HSP72, HIF-1a, HO-1, MMP-9, and other ischemia-related mediators, are

(A)

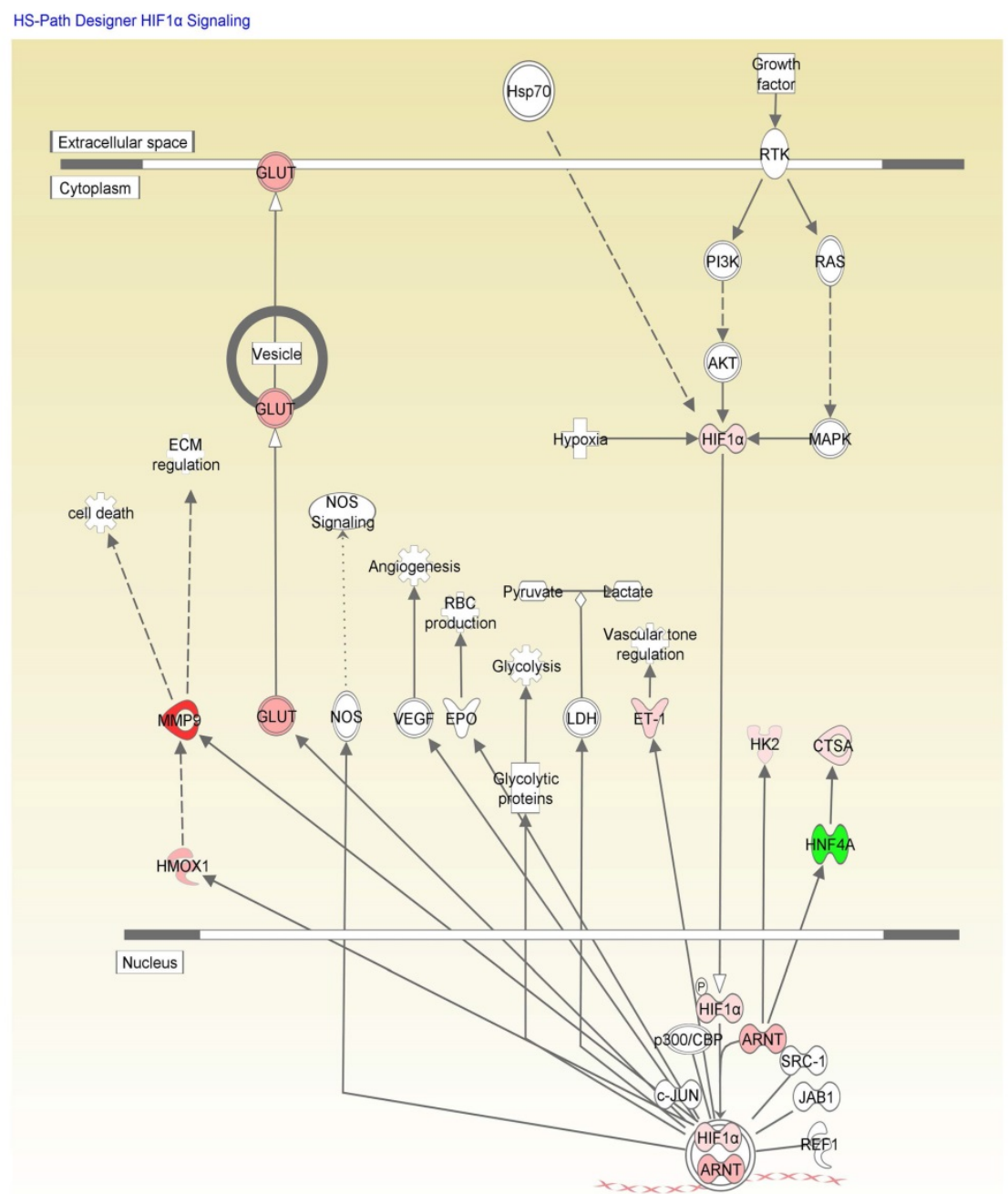

Q 2000-2014 OAAEN All rights reserved

(B)

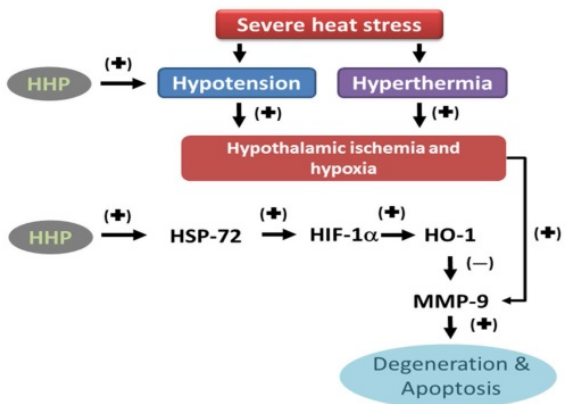

Figure 7. Core molecular net words identified by IPA. The altered genes are involved in biological functions associated with $\mathrm{HIF}$-1 $\alpha$ signaling (A). The red nodes and lines indicate the heat shock protein (HSP)70/HIF-1a/HMOX1 (or HO-1)/MMP-9 cell death pathway might be involved in the present results. (B) A flowchart of the proposed pathogenic mechanism of heatstroke in rats. Rodents subjected to heatstroke (evidenced by excessive hyperthermia and hypotension) exhibit higher levels of cellular ischemia and damage markers (e.g., MMP-9) in the hypothalamus, which leads to hypothalamic neuronal degeneration and apoptosis (I). Based on the core molecular net words identified by IPA shown in (B), HHP may attenuate heat-induced hypothalamic degeneration and apoptosis via the HSP70 (or HSP72)/HIF-1 a/HO-1/MMP-9 pathways (II). (+), upregulation; $(-)$ downregulation. HHP might ameliorate hypothalamic overexpression of MMP-9 in heat-exposed animals by reducing hypotension (or increasing CBF) and/or by enhancing HSP-72/HIF-1 $\alpha / \mathrm{HO}-1$ signaling. 
involved in significant biological functions associated with HIF-1a. Severe heat stress may cause both hypotension and hyperthermia and result in hypothalamic ischemic/hypoxia and overexpression of MMP-9, leading to hypothalamic degeneration and apoptosis (Fig. 7B). HHP may attenuate heat-induced hypothalamic degeneration and apoptosis by inhibiting the pathways involved in hypotension, hypothalamic ischemic and hypothalamic MMP-9 overexpression or enhancing the HSP-72/HIF-a/ HO-1/MMP-9 pathway.

\section{Limitations}

This study used adult male rats and therefore cannot reflect the complicated human clinical situation, which may involve different comorbidities. Male rats were used to avoid the hormonal fluctuations that occur in female rats. Additionally, in this study, thermal experiments were performed in rats under general anesthesia.

\section{Conclusions}

Based on our present data, we conclude that severe heat stress causes hypothalamic ischemia/ hypoxia, upregulation of hypothalamic MMP-9, and hypothalamic neuronal degeneration and apoptosis. HHP upregulates the hypothalamic protein expression of HSP-72, HIF-1a, and HO-1 and downregulates hypothalamic MMP-9 activity to attenuate hypothalamic neuronal degeneration and apoptosis in heat-exposed animals.

\section{Abbreviations}

HIF-1a: Hypoxia-inducible factor-1a; HSP-72: Heat shock protein-72; HO-1: Hemeoxygenase-1; MMP-9: Matrix metalloproteinase-9; HHP: hypobaric hypoxia preconditioning; CBF: cerebral blood flow; $\mathrm{PO}_{2}$ : partial oxygen pressure; Ta: ambient temperature; Tco: colon temperature; MABP: mean arterial blood pressure; HR: heart rate; NeuN: neuronal nuclei; Caspase-3: Cysteine-aspartic acid protease 3; DAPI: 4',6-Diamidino-2-phenylindole; IPA: ingenuity pathway analysis; BBB: blood-brain barrier; $3 \mathrm{~V}$ : third ventricle; ME: median eminence.

\section{Acknowledgments}

The authors appreciate Miss Mon-Tsung Ho for her excellent technical and executive assistance.

\section{Author Contributions}

C.M.C., C.H.L., C.P.C. and C.L.C. were involved in the collection, analysis, and interpretation of the data and manuscript writing and approval. K.C.N. L.Y.T. and L.S.L. were involved in the conception of the study and discussion of the results. L.S.L. and
C.P.C. were involved in manuscript writing and submission.

\section{Funding}

Our study was supported by grants from the Ministry of Science and Technology of Taiwan (MOST107-2314-B-384-007-MY3), Chi Mei Medical Center (Taiwan) (nos. CMFHT1040530, CMFHT10504, and CLFHR10825, CMORA10904).

\section{Competing Interests}

The authors have declared that no competing interest exists.

\section{References}

1. Chen $\mathrm{SH}$, Lin MT, Chang CP. Ischemic and oxidative damage to the hypothalamus may be responsible for heat stroke. Curr Neuropharmacol. 2013; 11: 129-40.

2. Singh LP, Kapoor M, Singh SB. Heat: not black, not white. It's gray!!! J Basic Clin Physiol Pharmacol. 2013; 24: 209-24.

3. Leon LR, Bouchama A. Heat stroke. Compr Physiol. 2015; 5: 611-47.

4. Chauhan NR, Kapoor M, Prabha Singh L, et al. Heat stress-induced neuroinflammation and aberration in monoamine levels in hypothalamus are associated with temperature dysregulation. Neuroscience. 2017; 358: 79-92.

5. [Internet] QIAGEN. QIAGEN Redwood City, QIAGEN'S Ingenuity Analysis 2015. [cited 2015; Available from: https://www.qiagenbioinformatics.com/ products/ingenuity-pathway-analysis/].

6. Chen ZC, Wu WS, Lin MT, et al. Protective effect of transgenic expression of porcine heat shock protein 70 on hypothalamic ischemic and oxidative damage in a mouse model of heatstroke. BMC Neurosci. 2009; 10: 111.

7. Chaturvedi M, Kaczmarek L. Mmp-9 inhibition: a therapeutic strategy in ischemic stroke. Mol Neurobiol. 2014; 49: 563-73.

8. Sharp FR, Zhan X, Liu DZ. Heat shock proteins in the brain: role of Hsp70, Hsp 27, and HO-1 (Hsp32) and their therapeutic potential. Transl Stroke Res. 2013; 4: 685-92.

9. Liu FJ, Kaur P, Karolina DS, et al. MiR-335 Regulates Hif-1alpha to Reduce Cell Death in Both Mouse Cell Line and Rat Ischemic Models. PLoS One. 2015; 10: e0128432.

10. Bisgard GE. Carotid body mechanisms in acclimatization to hypoxia. Respir Physiol. 2000; 121: 237-46.

11. Ely BR, Lovering AT, Horowitz M, et al. Heat acclimation and cross tolerance to hypoxia: Bridging the gap between cellular and systemic responses. Temperature (Austin, Tex.). 2014; 1: 107-14.

12. Samoilov MO, Lazarevich EV, Semenov DG, et al. The adaptive effects of hypoxic preconditioning of brain neurons. Neurosci Behav Physiol. 2003; 33: $1-11$.

13. Sharp FR, Ran R, Lu A, et al. Hypoxic preconditioning protects against ischemic brain injury. NeuroRx. 2004; 1: 26-35.

14. Gidday JM, Fitzgibbons JC, Shah AR, et al. Neuroprotection from ischemic brain injury by hypoxic preconditioning in the neonatal rat. Neurosci Lett. 1994; 168: 221-4.

15. Chen $\mathrm{CM}$, Hou $\mathrm{CC}$, Cheng $\mathrm{KC}$, et al. Activated protein $\mathrm{C}$ therapy in a rat heat stroke model. Crit Care Med. 2006; 34: 1960-6.

16. Council NR. Guide for the Care and Use of Laboratory Animals: Eighth Edition. 2011; Washington, DC: The National Academies Press. 246.

17. Chou YT, Lai ST, Lee CC, et al. Hypothermia attenuates circulatory shock and cerebral ischemia in experimental heatstroke. Shock. 2003; 19: 388-93.

18. Lin HJ, Wang $\mathrm{CT}$, Niu $\mathrm{KC}$, et al. Hypobaric hypoxia preconditioning attenuates acute lung injury during high-altitude exposure in rats via up-regulating heat-shock protein 70. Clin Sci (Lond). 2011; 121: 223-31.

19. Hsi-Hsing $Y$, Ching-Ping C, Juei-Tang C, et al. Inhibition of acute lung inflammation and injury is a target of brain cooling after heatstroke injury. J Trauma. 2010; 69: 805-12.

20. He $Y$, Hua $Y$, Liu $W$, et al. Effects of cerebral ischemia on neuronal hemoglobin. Journal of cerebral blood flow and metabolism : official journal of the International Society of Cerebral Blood Flow and Metabolism. 2009; 29: 596-605.

21. Banjara M, Ghosh C, Dadas A, et al. Detection of brain-directed autoantibodies in the serum of non-small cell lung cancer patients. PLOS ONE. 2017; 12: $\mathrm{e} 0181409$.

22. Asahi M, Asahi K, Jung JC, et al. Role for matrix metalloproteinase 9 after focal cerebral ischemia: effects of gene knockout and enzyme inhibition with BB-94. J Cereb Blood Flow Metab. 2000; 20: 1681-9.

23. Arai K, Lee SR, Lo EH. Essential role for ERK mitogen-activated protein kinase in matrix metalloproteinase-9 regulation in rat cortical astrocytes. Glia. 2003; 43: 254-64 
24. Honorio JER, Vasconcelos GS, Rodrigues FTS, et al. Monocrotaline: Histological Damage and Oxidant Activity in Brain Areas of Mice. Oxidative Medicine and Cellular Longevity. 2012;

25. Paxinos G, Watson C. The Rat Brain in Stereotaxic Coordinates. 7th ed. 2013; Academic Press.

26. Tuor UI, Zhao ZH, Barber PA, et al. Recurrent mild cerebral ischemia: enhanced brain injury following acute compared to subacute recurrence in the rat. Bmc Neuroscience. 2016; 17:

27. Honorio JER, Vasconcelos GS, Rodrigues FTS, et al. Monocrotaline: Histological Damage and Oxidant Activity in Brain Areas of Mice. Oxidative Medicine and Cellular Longevity. 2012; 2012.

28. Kaczmarek L. Mmp-9 inhibitors in the brain: can old bullets shoot new targets? Curr Pharm Des. 2013; 19: 1085-9.

29. Horowitz M. Matching the heart to heat-induced circulatory load: heat-acclimatory responses. News Physiol Sci. 2003; 18: 215-21.

30. Horowitz M. Heat acclimation and cross-tolerance against novel stressors: genomic-physiological linkage. Prog Brain Res. 2007; 162: 373-92.

31. Horowitz $M$, Assadi $H$. Heat acclimation-mediated cross-tolerance in cardioprotection: do HSP70 and HIF-1alpha play a role? Ann N Y Acad Sci. 2010; 1188: 199-206.

32. Alexander-Shani R, Mreisat A, Smeir E, et al. Long-term HIF-1alpha transcriptional activation is essential for heat-acclimation-mediated cross tolerance: mitochondrial target genes. Am J Physiol Regul Integr Comp Physiol. 2017; 312: R753-r62.

33. Maloyan A, Palmon A, Horowitz M. Heat acclimation increases the basal HSP72 level and alters its production dynamics during heat stress. Am J Physiol. 1999; 276: R1506-15.

34. Helton R, Cui J, Scheel JR, et al. Brain-specific knock-out of hypoxia-inducible factor-1alpha reduces rather than increases hypoxic-ischemic damage. J Neurosci. 2005; 25: 4099-107.

35. Chen $\mathrm{C}, \mathrm{Hu} \mathrm{O}$, Yan J, et al. Early inhibition of HIF-1alpha with small interfering RNA reduces ischemic-reperfused brain injury in rats. Neurobiol Dis. 2009; 33: 509-17.

36. Li Y, Zhou C, Calvert JW, et al. Multiple effects of hyperbaric oxygen on the expression of HIF-1 alpha and apoptotic genes in a global ischemia-hypotension rat model. Exp Neurol. 2005; 191: 198-210.

37. Turturici G, Sconzo G, Geraci F. Hsp70 and its molecular role in nervous system diseases. Biochemistry research international. 2011; 2011: 618127-27.

38. Hoshino T, Murao N, Namba $\mathrm{T}$, et al. Suppression of Alzheimer's disease-related phenotypes by expression of heat shock protein 70 in mice. The Journal of neuroscience : the official journal of the Society for Neuroscience. 2011; 31: 5225-34.

39. Yang CH, Yen TL, Hsu CY, et al. Multi-Targeting Andrographolide, a Novel NF-kappaB Inhibitor, as a Potential Therapeutic Agent for Stroke. Int J Mol Sci. 2017; 18:

40. Kim YM, Pae HO, Park JE, et al. Heme oxygenase in the regulation of vascular biology: from molecular mechanisms to therapeutic opportunities. Antioxid Redox Signal. 2011; 14: 137-67.

41. Ding $Y$, Chen M, Wang M, et al. Posttreatment with 11-Keto-beta-Boswellic Acid Ameliorates Cerebral Ischemia-Reperfusion Injury: Nrf2/HO-1 Pathway as a Potential Mechanism. Mol Neurobiol. 2015; 52: 1430-39.

42. Huang $Y, Y u$ J, Wan F, et al. Panaxatriol saponins attenuated oxygen-glucose deprivation injury in PC12 cells via activation of PI3K/Akt and Nrf2 signaling pathway. Oxid Med Cell Longev. 2014; 2014: 978034.

43. Piantadosi CA, Withers CM, Bartz RR, et al. Heme oxygenase-1 couples activation of mitochondrial biogenesis to anti-inflammatory cytokine expression. J Biol Chem. 2011; 286: 16374-85.

44. Opdenakker G, Van den Steen PE, Dubois B, et al. Gelatinase B functions as regulator and effector in leukocyte biology. J Leukoc Biol. 2001; 69: 851-9.

45. Dubois B, Starckx S, Pagenstecher A, et al. Gelatinase B deficiency protects against endotoxin shock. Eur J Immunol. 2002; 32: 2163-71.

46. Vu TH, Shipley JM, Bergers G, et al. MMP-9/gelatinase B is a key regulator of growth plate angiogenesis and apoptosis of hypertrophic chondrocytes. Cell. 1998; 93: 411-22.

47. Rivera S, Khrestchatisky M, Kaczmarek L, et al. Metzincin proteases and their inhibitors: foes or friends in nervous system physiology? J Neurosci. 2010; 30: 15337-57.

48. Rybakowski JK. Matrix Metalloproteinase-9 (MMP9)-A Mediating Enzyme in Cardiovascular Disease, Cancer, and Neuropsychiatric Disorders. Cardiovasc Psychiatry Neurol. 2009; 2009: 904836.

49. Gaspar JM, Velloso LA. Hypoxia Inducible Factor as a Central Regulator of Metabolism - Implications for the Development of Obesity. Front Neurosci. 2018; 12: 813.

50. Martinez D, Vasconcellos LF, de Oliveira PG, et al. Weight loss and brown adipose tissue reduction in rat model of sleep apnea. Lipids Health Dis. 2008; 7: 26

51. Suzuki D, Murata Y, Oda S. Changes in Ucp1, D2 (Dio2) and Glut4 (Slc2a4) mRNA expression in response to short-term cold exposure in the house musk shrew (Suncus murinus). Exp Anim. 2007; 56: 279-88. 